\title{
PASSAGE À L'ÂGE ADULTE, HANDICAP ET CONFIGURATIONS INCLUSIVES
}

\author{
Serge Ébersold, Philippe Cordazzo
}

Presses de Sciences Po (P.F.N.S.P.) | «Agora débats/jeunesses »

2015/3 Nº 71 | pages 55 à 67

ISSN 1268-5666

ISBN 9782724633917

Article disponible en ligne à l'adresse :

http://www.cairn.info/revue-agora-debats-jeunesses-2015-3-page-55.htm

\section{Pour citer cet article :}

Serge Ébersold, Philippe Cordazzo, « Passage à l'âge adulte, handicap et configurations inclusives », Agora débats/jeunesses 2015/3 (N 71), p. 55-67. DOI 10.3917/agora.071.0055

Distribution électronique Cairn.info pour Presses de Sciences Po (P.F.N.S.P.).

(C) Presses de Sciences Po (P.F.N.S.P.). Tous droits réservés pour tous pays.

La reproduction ou représentation de cet article, notamment par photocopie, n'est autorisée que dans les limites des conditions générales d'utilisation du site ou, le cas échéant, des conditions générales de la licence souscrite par votre établissement. Toute autre reproduction ou représentation, en tout ou partie, sous quelque forme et de quelque manière que ce soit, est interdite sauf accord préalable et écrit de l'éditeur, en dehors des cas prévus par la législation en vigueur en France. Il est précisé que son stockage dans une base de données est également interdit. 


\section{Passage à l'âge adulte, handicap et configurations inclusives}

Serge Ebersold, Philippe Cordazzo

$\mathrm{L}$

a jeunesse est une période de la vie jalonnée de seuils statutaires (départ du foyer parental, mise en couple, fin des études, etc.) à partir desquels se construit la position sociale. La poursuite d'études après le baccalauréat requiert une distanciation vis-à-vis de la figure de l'élève, dont la scolarité est prescrite et très encadrée, au profit de celle de l'étudiant, relativement libre de ses choix et de son niveau d'investissement dans le cursus. La confrontation au marché de l'emploi suppose une projection de soi dans le temps et force ainsi à se tourner vers un passé qu'il faut assumer et un futur qu'il faut envisager, malgré de multiples incertitudes. L'éloignement familial préfigure l'installation dans une vie de couple et confronte le jeune adulte à de nouvelles exigences et à de nouveaux rôles sociaux, surtout quand survient la naissance d'enfants.

Ces seuils statutaires connaissent toutefois une certaine dilution qui renouvelle fondamentalement les conditions sociales et psychiques d'entrée dans la vie adulte (Van de Velde, 2008). Le chômage d'insertion retarde l'entrée sur le marché du travail et renforce le risque de marginalisation, en particulier en cas de sous-qualification. La précarisation de l'emploi multiplie les périodes de transition, diversifie les trajectoires d'insertion et accroît le degré d'exposition aux risques de disqualification qui entourent le chômage récurrent. L'augmentation du travail à temps partiel précarise socialement et financièrement par des salaires souvent inférieurs à la moyenne, des prestations sociales moindres et des perspectives professionnelles plus limitées (OCDE, 2010). Enfin, l'allongement des études, la maîtrise de la fécondité et l'affirmation d'une vie de couple sans enfants ont contribué à isoler la naissance du premier enfant des autres composantes du passage à l'âge adulte (Cordazzo, Fichet, 2013). Ces évolutions ont affaibli le poids de l'âge comme hiérarchie de classement, retardé l'entrée dans la vie professionnelle et dans la vie familiale et, corrélativement, contribué à une déritualisation du passage à l'âge adulte. Celle-ci a transformé cette période de la vie en un moment où prévaut l'expérimentation de parcours construits au gré d'un processus itératif fait d'essais et d'erreurs, et où la mobilité individuelle, la 


\section{Dossier}

Passage à l'âge adulte, handicap

et configurations inclusives

capacité de choix, le sens de l'expérimentation et un rapport positif au futur sont au cœur du processus de transition (Galland, 2011).

Ce modèle de l'expérimentation est encore plus marqué pour les jeunes présentant un besoin éducatif particulier (BEP). Les critères d'âge changent les approches du handicap et bouleversent les conditions d'éligibilité aux aides et aux aménagements : dans nombre de pays, comme par exemple la Norvège, l'approche du handicap n'est plus rapportée aux normes scolaires, mais à celles régissant le monde de l'emploi. Le caractère interinstitutionnel du traitement social du handicap interroge la continuité et la cohérence des parcours à l'issue de l'enseignement secondaire à l'intrication de plusieurs dimensions : celles liées à l'accessibilité relevant de l'institution scolaire ; celles liées à la compensation de la déficience sous l'égide du ministère des affaires sociales; et celles relevant des instances locales comme les transports ou le logement.

Ces particularités surexposent les jeunes adultes présentant un besoin éducatif particulier à des ruptures de parcours qui les confrontent de manière croissante au chômage de longue durée et à la pauvreté (OCDE, 2010). Ceux qui ne se jugent pas handicapés ou qui se refusent à signaler leur particularité lors de leur inscription dans l'enseignement supérieur ou lors d'un recrutement, par crainte d'être stigmatisés, se trouvent privés de leurs droits (Newman et al., 2009). Le réamorçage de démarches souvent longues, coûteuses en temps et en énergie transforment les dimensions régissant la poursuite de la scolarité ou l'accès à l'emploi en évènements révélateurs de handicap qui opère un véritable travail d'invalidation. À l'image d'une râpe, ces discontinuités érodent les représentations que les individus se font d'eux-mêmes et transforment la volonté d'appartenir et d'exister en un facteur de désenchantement et de déréalisation de soi qui retire la possibilité de se penser d'égale valeur par rapport aux autres (Ebersold, 2007). Elles rendent les individus vulnérables dans le rapport qu'ils entretiennent avec eux-mêmes, les privent de la confiance en soi et du respect de soi qui leur permettraient de se considérer sujet de leur devenir. Elles font partie intégrante du processus de production du handicap auquel sont exposés les jeunes adultes à besoins éducatifs particuliers.

Cet article s'intéressera aux formes d'affiliation expérimentées par ces derniers à l'issue du lycée. Celles-ci résident dans le degré d'indépendance économique et sociale grâce à laquelle les intéressés accèdent aux mêmes rôles sociaux que tout un chacun et inscrivent les échanges dans une dynamique de réciprocité. Elles s'organisent également autour du capital identitaire leur permettant d'être confiants et conscients des attentes qui les concernent et de s'estimer capables de faire face aux changements et aux exigences qui peuvent y être liés (Côté, 1996). Elles reposent en outre sur l'autonomie décisionnelle. Cet effet d'affiliation réside enfin dans le sentiment d'exister qu'éprouvent les jeunes adultes à besoins éducatifs particuliers au contact d'autrui, notamment 


\section{Dossier \\ Passage à l'âge adulte, handicap et configurations inclusives}

des professionnels, et que peuvent révéler la qualité de vie qu'ils se perçoivent ainsi que la possibilité de se penser et d'être pensés aussi respectables et estimables que tout un chacun (Flahaut, 2002). Cette perspective enrichit le concept d'affiliation proposé par Robert Castel (1995) par l'inclusion des dimensions symboliques et identitaires dans le pacte social autour duquel se joue l'incorporation durable des individus dans un ordre social garanti par l'État. Elle corrèle les mécanismes intervenant dans le passage à l'âge adulte à l'interpénétration de facteurs objectifs conditionnant l'égalité juridique, l'indépendance économique et sociale et de facteurs intersubjectifs assurant une égalité des chances dans la recherche de l'estime sociale (Fraser, 2005 ).

\section{Méthodologie}

Cet article s'appuie sur une enquête réalisée en 2009 auprès de 293 lycéens à besoins éducatifs particuliers (BEP) vivant en France et inscrits en classe de terminale en 2006-2007, à l'aide d'un questionnaire bâti dans le cadre d'une recherche menée par l'OCDE sur les parcours des jeunes adultes à BEP dans cinq pays membres (Danemark, France, République tchèque, Norvège, Pays-Bas). 70 entretiens semi-directifs ont également été menés avec des jeunes adultes à BEP ainsi qu'avec des personnes ayant joué, selon eux, un rôle important dans leurs parcours (Rick, Ebersold, 2011 ; Ebersold, 2012).

Le questionnaire ainsi que les grilles d'entretiens ont été élaborés conjointement par des représentants des pays participants selon cinq descripteurs : le descripteur de participation considère l'activité exercée à l'issue du lycée (formes de participation, conditions de participation offertes, adéquation aux attentes et aux profils des intéressés); (e descripteur d'accessibilité porte le regard sur les aménagements et soutiens existants, leur adéquation aux besoins des intéressés et leur aptitude à les placer à égalité de chances avec leurs pairs; le descripteur de performance se focalise sur la réussite scolaire, la préparation à la vie professionnelle ou la poursuite des études; le descripteur de transition interroge la continuité et la cohérence des parcours suivis, les soutiens et les stratégies développées en la matière ; le descripteur d'affiliation concerne enfin le niveau d'indépendance économique et sociale des intéressés, leur capacité de projection dans le futur, leur capital identitaire (Côté, 1996), ainsi que leur sentiment d'appartenance.

L'analyse s'organise ainsi autour des éléments fournis par les traitements univariés et bivariés des données ainsi que par l'étude des correspondances multiples (ACM) pour laquelle a été notamment mobilisée la méthode de la classification ascendante hiérarchique (CAH) qui a permis de créer des variables synthétiques regroupant plusieurs indicateurs. Ces données sont complétées par des extraits d'entretiens réalisés lors des études de cas. Le texte décrit ici, dans un premier temps, les situations des jeunes à BEP interrogés à l'issue de la terminale ainsi que leur effet d'affiliation pour, dans un second temps, relier les formes d'affiliation expérimentées aux dynamiques régissant les trajectoires scolaires ainsi que la transition vers l'enseignement supérieur à l'issue du lycée. Il relie en dernier lieu les dynamiques d'affiliation révélées par l'analyse aux stratégies d'accompagnement développées en terminale. 
Dossier

Passage à l'âge adulte, handicap

et configurations inclusives

\section{LES JEUNES ADULTES À BESOINS ÉDUCATIFS PARTICULIERS INÉGAUX EN TERMES DE DEVENIR ET D'AFFILIATION}

Une grande majorité des jeunes adultes à besoins éducatifs particuliers enquêtés (78\%) poursuivent leurs études à l'issue du lycée. La quasi-totalité d'entre eux est inscrite dans un établissement d'enseignement supérieur tout comme le reste des jeunes lycéens qui continuent leurs études. Cette poursuite d'études n'est cependant pas toujours source d'indépendance : seuls $22 \%$ déclarent être financièrement autonomes et leurs revenus proviennent rarement de salaires à la différence de l'ensemble des jeunes âgés de 18 à 24 ans (CESE, 2009). Ils sont également moins nombreux que les autres à avoir quitté leur famille : $63 \%$ des jeunes à besoins éducatifs particuliers vivent encore au domicile familial contre $56 \%$ de l'ensemble des 18 à 29 ans (Pan Ké Shon, 2010). La décohabitation familiale est entravée par l'absence de permis de conduire lévoquée par $39 \%$ des enquêtés), par l'inaccessibilité des transports publics, par un accès plus restreint au logement et à l'emprunt : $48 \%$ des enquêtés jugent ne pas avoir les mêmes possibilités d'emprunt que les autres jeunes et se trouvent dépendants des aides financières allouées par la famille (principale ressource financière pour $56 \%$ des enquêtés) ou les services publics (principale ressource financière pour $25 \%$ d'entre eux). Au-delà des dimensions spécifiques à la déficience ou à l'état de santé des enquêtés ${ }^{1}$, ce manque d'indépendance les prive de la réactivité requise par la multiplication des périodes de transition et la désynchronisation des étapes jalonnant l'entrée dans la vie adulte. L'exercice d'une activité requiert souvent une identification du degré d'accessibilité des lieux, des soutiens nécessaires et des aides disponibles et suppose, conséquemment, un travail de planification peu propice à la spontanéité ou à la réactivité (Ebersold, 2006).

\section{DES POSSIBILITÉS D’AFFILIATION SUBORDONNÉES À UNE MISE EN SCÈNE DE SOI}

Ces possibilités d'affiliation dépendent aussi des conditions de scolarité, du sentiment d'appartenance induit par la trajectoire scolaire et de la position occupée à l'issue du lycée. Le premier facteur de l'analyse factorielle (contribution $30,4 \%$ sur le graphique 1, p. 59) oppose les jeunes adultes ayant accédé à l'enseignement supérieur (extrémité gauche) à ceux qui ont arrêté leurs études à la fin du lycée (extrémité droite). Les enquêtés ayant accédé à l'enseignement supérieur jugent leur autonomie et leur qualité de vie satisfaisantes et estiment être aussi respectés et respectables que tout

1. Le type de déficience n'est pas statistiquement discriminant : les enquêtés estiment d'une manière générale leur état de santé excellent et jugent dans leur grande majorité n'avoir besoin d'aucune aide pour effectuer les tâches de la vie quotidienne (85\%), se déplacer (90\%), communiquer avec autrui (90\%), prendre soin d'eux-mêmes (93\%) ou effectuer les tâches ménagères (88\%). 
Graphique 1. Devenir des lycéens à besoins éducatifs particuliers inscrits en terminale en $\mathbf{2 0 0 7}$ et formes d'affiliation expérimentées

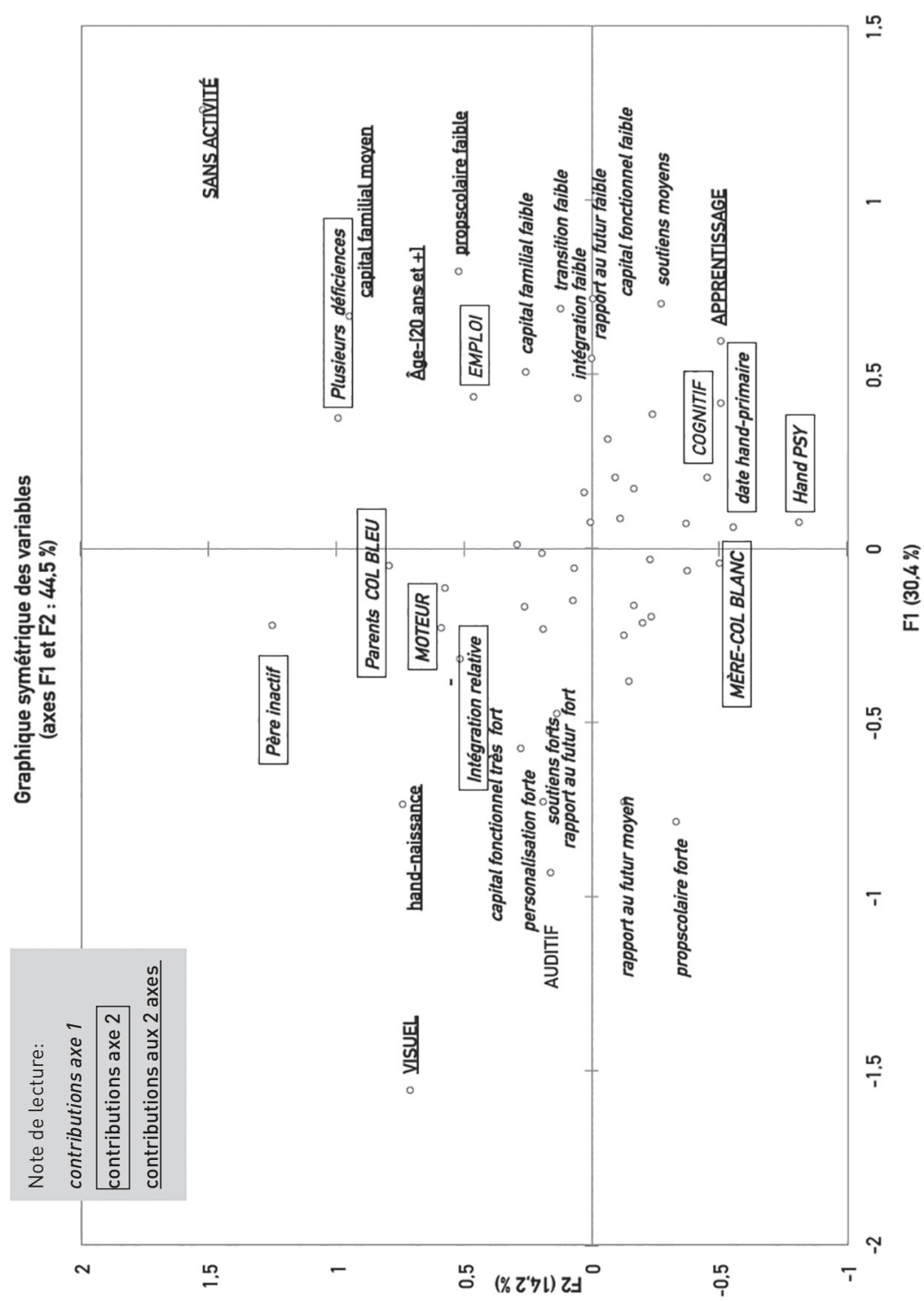

Note : chaque modalité des variables est représentée selon les coordonnées principales dans l'axe 1 et 2. Par souci de lisibilité, seules sont représentées les variables contributives et dont le cosinus est élevé (en valeur absolue) confirmant ainsi que la variable est fortement liée à l'axe.

Champ : enquête portée en 2009 auprès de 293 lycéens français à besoins éducatifs particuliers, inscrits en terminale en 2006-2007. 


\section{Dossier}

Passage à l'âge adulte, handicap

et configurations inclusives

un chacun. Ils disposent d'un capital scolaire favorisant l'accès à l'enseignement supérieur : titulaires d'un baccalauréat général et n'ayant pas redoublé, leur transition vers l'enseignement supérieur n'a pas été obérée par un retard scolaire, comme c'est souvent le cas en France. Présentant le plus souvent une déficience survenue à la naissance, ils relient leur réussite à la qualité des aménagements et des soutiens dont ils ont bénéficié en terminale lélaboration de projets personnalisés de scolarisation fondés sur une analyse des besoins, flexibilité des pratiques pédagogiques et engagement des enseignants, existence d'un suivi tout au long de l'année scolaire). Leur trajectoire à l'issue du lycée est également à relier au capital identitaire (Côté, 1996) acquis au cours de la scolarisation : ils estiment avoir été préparés aux

Les enquêtés ayant accédé à l'enseignement supérieur jugent leur autonomie et leur qualité de vie satisfaisantes et estiment être aussi respectés et respectables que tout un chacun. rôles sociaux liés à l'accès à l'enseignement supérieur et être à même de faire valoir leurs droits; ils pensent que l'école les a également préparés à s'affirmer, sensibilisés à leurs besoins, conduits à identifier leurs points forts et à avoir confiance dans leurs décisions. Cette trajectoire résulte toutefois aussi du « capital familial » qu'ont pu mobiliser certains intéressés indiquant avoir été soutenus scolairement, techniquement et financièrement par leur famille. Celle-ci a, notamment, pallié les dysfonctionnements institutionnels durant la scolarité, veillé à la continuité des parcours lors des périodes de transition ou encore facilité l'obtention d'un logement.

Ces jeunes adultes inscrits dans l'enseignement supérieur se distinguent des inactifs ou de ceux exerçant un emploi au moment de l'enquête. Ces derniers ont été exposés à la ségrégation scolaire et leurs parcours scolaires sont plus chaotiques : plus âgés que la moyenne, ils ont été confrontés aux logiques disqualifiantes de l'enseignement professionnel et ont connu des difficultés scolaires. Par ailleurs, ils sont plus enclins que la moyenne à penser ne pas être en mesure de satisfaire aux exigences du marché de l'emploi ou de l'enseignement supérieur. Volontiers critiques sur le déroulement de leur scolarité, ces jeunes adultes regrettent que celle-ci n'ait pas été structurée, ainsi qu'y invitent les textes, autour d'un projet personnalisé de scolarisation fondé sur une évaluation des besoins. Ils minimisent le rôle des soutiens dans leur réussite, estimant ne pas avoir progressé au même rythme que les autres élèves : selon eux, ces soutiens ne leur ont pas permis de concilier leurs études avec les contraintes de la déficience, d'effectuer les mêmes tâches et activités que leurs camarades et, plus généralement, d'être à égalité de chances avec eux ; ils ne leur auraient pas permis en outre de se projeter dans le futur, de choisir leur cursus en connaissance de cause, voire de pallier l'absence de soutien familial. Plus largement, ils se disent 
exclus de la société, de moindre valeur sociale que les autres et déplorent ne pas avoir l'autonomie et la qualité de vie souhaitées; ils regrettent que leur situation ne leur offre pas une autonomie financière satisfaisante et ne soit pas source de reconnaissance ${ }^{2}$.

Si l'accès à l'enseignement supérieur a un tel effet d'affiliation, ce n'est pas le cas de l'accès à l'emploi, surtout s'il est exercé par défaut ou si la fonction occupée n'est pas satisfaisante. Le rapport à soi qu'entretiennent les enquêtés ayant accédé à l'enseignement supérieur atteste le capital symbolique qu' offre un niveau de qualification mettant à distance le lien ontologique qui unit l'altérité biologique à l'altérité sociale (Bourdieu, 1980). Véritable titre de noblesse au sein d'une population traditionnellement peu qualifiée ${ }^{3}$, le niveau de qualification opère un travail d'institution autorisant les intéressés à se penser différemment et à demander à être pensés différemment. Pour exemple, la moitié des enquêtés refusent de signaler leurs besoins éducatifs lors de leur inscription dans l'enseignement supérieur, surtout quand leur parcours scolaire les incite à ne pas se considérer handicapés. Un étudiant présentant un trouble de l'apprentissage estime ainsi avoir " plus de mérite que quelqu'un qui a eu le même parcours que [lui] et dans des situations à peu près similaires » et avoir su faire preuve d'aptitudes qui constituent « une surperformance [...] par rapport à une autre personne».

Ce travail d'institution est en cela indissociable de la mise en scène de soi exigée des élèves à besoins éducatifs particuliers dont la légitimité scolaire est reconnue ex post et non ex ante comme c'est pourtant le cas pour l'ensemble de la population. Selon les enquêtés, cette mise en scène de soi s'organise autour de leur détermination, de leur engagement et des ressources personnelles et familiales mobilisées pour faire face aux périodes critiques et surmonter les difficultés. Ils insistent sur l'importance de la confiance en soi, le courage et la combativité leur ayant permis d'agir en tant que facilitateurs, capables de compenser les dysfonctionnements, de contribuer activement au bon déroulement de leur scolarisation. Ils parlent de leur sens stratégique les incitant à faire appel, le cas échéant, à la fratrie, aux camarades, voire au personnel enseignant, pour pallier les lacunes occasionnées par les absences dues à la déficience, par le manque de soutiens appropriés ou encore par les insuffisances des enseignements.

2. Les jeunes inactifs sont plus dubitatifs que la moyenne des enquêtés sur leur capacité d'autodétermination ( $55 \%$ contre $81 \%$ ) et sont moins enclins à se juger aussi capables que les autres $(73,7 \%$ contre $88 \%)$. Ils estiment à l'inverse plus fréquemment se heurter à des obstacles lorsqu'ils cherchent à entreprendre quelque chose ( $47 \%$ contre $26 \%$ ) et disent plus fréquemment ( $37 \%$ contre $19 \%$ ) éviter de faire des projets, ces derniers n'aboutissant que trop rarement.

3. À titre indicatif, l'enquête Handicap-santé volet Ménages menée en 2008 révèle que seulement $6 \%$ des jeunes handicapés âgés de $20-24$ ans sont titulaires d'un diplôme de l'enseignement supérieur alors que cette proportion est de $29,6 \%$ dans l'ensemble de la population de même âge. 


\section{Dossier}

Passage à l'âge adulte, handicap

et configurations inclusives

Cet engagement personnel participe d'une mise en scène de l'identité scolaire opérant une inversion symbolique autour de laquelle se définit la légitimité scolaire. Cette mise en scène de l'identité scolaire permet à l'élève d'agir en tant que facilitateur compensant les dysfonctionnements liés aux contraintes organisationnelles et contribuant activement au bon déroulement de la scolarisation au même titre que les autres acteurs impliqués dans le processus. À la figure de l'élève en difficulté dépendant de l'institution scolaire, elle substitue celle de l'élève coconstructeur de son devenir. Les facultés d'adaptation ou les formes de compensation développées sont des signes révélateurs d'aptitudes et de qualités qui attestent une distanciation vis-à-vis de la passivité à l'égard des pratiques enseignantes et éducatives au profit de l'investissement de l'acteur stratège contribuant au consensus sur le sens de la scolarisation. Cette coproduction du sens de la scolarisation est mesurée à l'aune de l'explicitation des attentes et des besoins, de la possession des compétences scolaires, relationnelles et sociales facilitant l'inscription sociale et professionnelle de l'élève. Elle fait valoir ces composantes fondamentales de la légitimité scolaire que sont la bonne volonté, la docilité, la disponibilité, l'ardeur au travail (MENESR, 2008). Cette mise en scène répond à la conception méritocratique qui sous-tend l'accessibilisation de l'environnement scolaire et subordonne la différentiation des pratiques et la mise en œuvre des soutiens à la proximité des élèves avec les représentations dominantes du bon élève auprès des acteurs de l'école, surtout lorsque le processus de scolarisation n'a pas été légitimé de manière formelle et institutionnelle.

\section{DES POSSIBILITÉS D’AFFILIATION LIÉES AU CAPITAL COMPENSATOIRE POSSÉDÉ}

Subordonné à l'invention de soi des élèves, ce capital compensatoire s'organise également autour de l'engagement des parents. Le second facteur (contribution 14,2\% sur le graphique 1) relie en effet le devenir des jeunes interrogés et son effet d'affiliation à l'origine sociale des parents : il oppose les enquêtés d'origine modeste (extrémité supérieure) ayant connu une rupture à l'issue de l'enseignement secondaire à ceux provenant de milieux plus aisés qui ont accédé directement à l'enseignement supérieur et, dans une moindre mesure, à un emploi (extrémité inférieure). Ceux qui ne sont ni en emploi ni dans l'enseignement supérieur au moment de l'enquête sont proportionnellement plus âgés que la moyenne des enquêtés et ont un sentiment d'appartenance affaibli par une dépendance financière visà-vis de l'entourage familial. Leur passé scolaire a été marqué par un ou plusieurs redoublement(s) et ils présentent le plus souvent une déficience (par exemple des déficiences multiples ou une déficience motrice) ayant induit des absences répétées ou nécessitant la mobilisation d'une pluralité 


\section{Dossier \\ Passage à l'âge adulte, handicap et configurations inclusives}

d'agents pour articuler accessibilité et compensation. Ces difficultés scolaires sont moins imputées aux aménagements et aux soutiens scolaires qu'à une absence de stratégie en matière de transition qui leur donne l'impression d'être « mis à la porte du lycée et laissés tout seuls avec leur diplôme », notamment quand les enseignements et les soutiens sont jugés insuffisamment adaptés aux exigences de l'enseignement supérieur et du marché de l'emploi. Ce sentiment de solitude est renforcé par les difficultés à s'appuyer sur leur environnement familial ou amical pour compenser les lacunes des stratégies déployées par les lycées et les établissements d'enseignement supérieur en matière de transition et pour garantir une forme de continuité de parcours qui requiert bien souvent une bonne articulation des dimensions liées à l'accessibilité et à la compensation.

Les jeunes adultes à besoins éducatifs particuliers se situant à l'extrémité inférieure du second axe diffèrent sur bien des points.

Véritable titre de noblesse au sein d'une population traditionnellement peu qualifiée, le niveau de qualification opère un travail d'institution autorisant les intéressés à se penser différemment et à demander à être pensés différemment. Présentant le plus souvent un trouble de l'apprentissage ou un trouble psychique - que la confrontation et la distance aux normes d'apprentissage et de comportement scolaires ont le plus souvent « révélé »-, ils ont accédé à l'enseignement supérieur dans le prolongement de leur scolarité ; leur passé scolaire est peu heurté et leur progression scolaire correspond à celle des autres élèves. Critiques à l'égard des conditions de déroulement de celle-ci, ils jugent le niveau de personnalisation des pratiques et des soutiens insuffisant et attribuent à ces derniers un rôle mineur dans leur réussite scolaire et leur situation au moment de l'enquête. L'absence ou l'inefficacité des soutiens formels font du passage vers l'enseignement supérieur et vers l'emploi un parcours construit, selon la formule d'un interviewé, " avec des briques mais sans avoir de ciment » et compensé par l'investissement des parents dont les propriétés sociales ont permis d'assurer la continuité des cheminements et l'affiliation sociale des intéressés.

L'opposition sur le second facteur relie ainsi les trajectoires postscolaires à l'articulation des dimensions liées à l'accessibilité - qui sont sous la responsabilité des établissements scolaires et universitaires - et à celles renvoyant à la compensation des implications sociales de la déficience - qui relèvent du secteur social et médico-social. D’autant plus malaisée que la déficience est sévère, cette articulation dépend du volume et de la structure des ressources mobilisées par les parents pour créer les passerelles entre les espaces éducatifs et administratifs. Il est question des ressources financières nécessaires pour pallier les surcoûts liés au logement ou encore aux transports 


\section{Dossier}

Passage à l'âge adulte, handicap

et configurations inclusives

et de la mobilisation du cercle relationnel nécessaire à l'accès à l'emploi. Il est aussi question du capital culturel requis pour satisfaire à la complexité des exigences administratives et aux exigences d'une contractualisation des pratiques supposant des capacités de négociateur lors de la définition du projet ou des diverses réunions organisées en lien avec la scolarisation de l'enfant. Il est en outre question du « capital temps » rendu nécessaire par un système éducatif qui demande aux intéressés et à leurs parents de réunir les conditions de son accessibilité et de leur inscription sociale. Ces propriétés ont vocation à transformer l'environnement social en ressources susceptibles de soutenir l'école, de relativiser le lien entre incapacité et déficience, et d'atténuer le poids des représentations profanes qui peuvent entourer le handicap et

La transition vers l'enseignement supérieur est plus aisée quand les parents sont titulaires d'un diplôme d'enseignement supérieur alors que les parcours postscolaires sont plus heurtés quand ils sont d'origine modeste. la personne handicapée. Aussi la transition vers l'enseignement supérieur est-elle plus aisée quand les parents sont titulaires d'un diplôme d'enseignement supérieur alors que les parcours postscolaires sont plus heurtés quand ils sont d'origine modeste ${ }^{4}$.

Le système scolaire corrèle donc les possibilités d'affiliation des jeunes adultes à besoins éducatifs particuliers au capital compensatoire conféré par la capacité d'autolégitimation et d'autoprotection des intéressés et de leur entourage familial. Il les surexpose aux mécanismes sélectifs régissant l'accès à l'enseignement supérieur et à l'emploi, renforce le poids des préjugés à leur égard et, in fine, l'effet invalidant de la déficience. Il réduit par ailleurs la notion de réussite à sa stricte composante scolaire au détriment des diverses dimensions intervenant dans la mise en sens d'un avenir social inscrit dans les rôles sociaux constitutifs du passage à l'âge adulte. Il prive ainsi les jeunes adultes d'origine modeste d'un capital social collectif (Lecoutre, 2003) essentiel à l'élaboration des passerelles requises pour la continuité des parcours ${ }^{5}$. Le système scolaire confond de surcroît besoin éducatif et déficience et n'organise pas la scolarisation des élèves à besoins éducatifs particuliers autour d'un projet personnalisé de scolarisation, notamment lorsqu'ils présentent un trouble des apprentissages. Il ancre ainsi la personnalisation des pratiques et la mise en œuvre des soutiens dans une

4. L'analyse révèle que $21 \%$ des enfants de parents ouvriers ou employés exercent un emploi contre $8,5 \%$ des enfants de parents provenant de milieux plus aisés, alors que l'inactivité est plus fréquemment observable parmi celles et ceux dont les parents sont inactifs (11,9\%). Par ailleurs, les enquêtés ayant ces profils ont plus fréquemment mis plus de trois mois pour accéder à l'enseignement supérieur ou à l'emploi.

5. Les enquêtés dont la mère a accédé à l'enseignement supérieur et exerce des fonctions d'encadrement sont proportionnellement plus nombreux $(81,2 \%$ contre $57,5 \%)$ à avoir été soutenus par leur famille à l'issue du lycée et à juger cet impact important $(69,3 \%$ contre 48,5\%). 


\section{Dossier \\ Passage à l'âge adulte, handicap et configurations inclusives}

logique d'aide peu propice au changement des représentations entourant les difficultés scolaires, peu orientée vers l'acquisition des apprentissages et peu à même de permettre aux intéressés de concilier les exigences liées à la réussite scolaire avec celles imposées par la déficience. La scolarisation s'en trouve organisée autour d'un principe de servitude subordonnant l'accessibilité de l'environnement scolaire et la reconnaissance d'autrui à l'aptitude des intéressés et de leur famille à se rendre acceptables. Le principe d'égalité des chances est en cela soumis au degré de proximité des intéressés vis-à-vis des normes scolaires et les inégalités entre les jeunes adultes présentant un besoin éducatif particulier et la population totale s'en trouvent renforcées : les enquêtés les moins enclins à se juger partie prenante de la société et les plus pessimistes à propos de leur avenir sont issus de milieux modestes ${ }^{6}$.

\section{ÉLÉMENTS DE CONCLUSION}

Les jeunes adultes à besoins éducatifs particuliers sont surexposés aux vulnérabilités qu'engendrent les difficultés d'accès à l'enseignement supérieur et à l'emploi, et les politiques développées ces dernières décennies n'ont pas contribué à réduire les inégalités les séparant de l'ensemble de la population, bien au contraire (OCDE, 2010). Cette surexposition trouve sa source dans une inaccessibilité les confinant dans une vie programmatique contraire à l'exigence croissante de mobilité et perpétuant leur dépendance à l'entourage familial. Elle résulte des inégalités scolaires dont est porteur un système éducatif qui conditionne la réussite scolaire à un surinvestissement en temps et en énergie de l'élève et de sa famille. Cette exigence d'engagement induit un retard scolaire, en même temps qu'elle est productrice d'inégalités sociales et scolaires; elle résume en outre le devenir de l'élève à l'obtention d'un diplôme, au détriment des dimensions conditionnant son inscription universitaire, professionnelle et sociale. Cette surexposition aux inégalités résulte aussi d'une exigence d'autolégitimation qui contraint les trajectoires scolaires à la possession d'un capital compensatoire permettant aux élèves d'être les coconstructeurs du processus, de rendre accessible l'environnement éducatif. Un tel capital opère l'inversion symbolique nécessaire à la légitimation scolaire des lycéens au regard des pratiques enseignantes, de la mobilisation des aménagements et des soutiens requis pour la scolarisation. Source d'inégalités, cette exigence d'autolégitimation et d'autoprotection fait aussi de l'engagement une source de désenchantement et de la scolarité un révélateur de handicap qui participe au processus de production de handicap.

6. La surreprésentation ouvrière observable dans les établissements pour enfants ou adultes handicapés atteste cela également, puisque les enfants de familles ouvrières sont sept fois plus souvent pensionnaires d'un établissement pour enfants et adolescents que ceux des cadres ; mais cette surreprésentation est encore plus importante lorsque les personnes sont adultes, et douze ou treize fois plus élevée pour les ouvriers que pour le reste de la population (Mormiche, 1999). 
Dossier

Passage à l'âge adulte, handicap

et configurations inclusives

\section{BIBLIOGRAPHIE}

Bourdieu P., Le sens pratique, Minuit, coll. « Le sens commun », Paris, 1980.

CAstel R., les métamorphoses de la question sociale, Fayard, Paris, 1995.

Conseil économique, social et environnemental (CESE), L'insertion des jeunes, CESE, Paris, 2009.

CôtÉ J., « Sociological perspectives of identity formation : the cultureidentity link and identity capital », Journal of Adolescence, $n^{\circ} 5$, vol. XIX, octobre 1996, p. 417-428.

Cordazzo P., Fichet B. (dir.), Transition, passage en sciences sociales, Néothèque, Strasbourg, 2013.

Ebersold S., « La participation à l'aune de l'affiliation », Université de Strasbourg (doc. ronéoté), Strasbourg, 2006.

Ebersold S., Parents et professionnels face au dévoilement du handicap, Érès, coll. « Connaissances de la diversité », Toulouse, 2007.

Ebersold S., Les transitions vers l'enseignement tertiaire et l'emploi pour les jeunes handicapés, OCDE, coll. «Politiques d'éducation et de formation », Paris, 2012.

Flahaut F., Le sentiment d'exister, Descartes et Cie, Paris, 2002.

Fraser N., Qu'est ce que la justice sociale ? Reconnaissance et redistribution, La Découverte, Paris, 2005.

Galland 0., Sociologie de la jeunesse, Armand Colin, coll. «U », Paris, 2011.

Lecoutre M., « Le capital social dans les transitions entre l'école et l'entreprise », in Bevort A., Lallement M., Le capital social. Performance, équité et réciprocité, La Découverte/Mauss, Paris, 2003.

Ministère de L'Éducation nationale, de L'enseignement supérieUR ET dE LA RECHERCHE (MENESR), Repères \& références statistiques sur les enseignements, la formation et la recherche (RERS 2008), MENESR/DEPP, Paris, 2008.

MoRmiche P., « Les personnes dépendantes en institution », INSEE première, n 669, août 1999.

Newman L., Wagner M., Cameto R. et Al., The Post-High School Outcomes of Youth With Disabilities up to 4 Years After High School. A Report From the National Longitudinal Transition Study-2 (NLTS2), SRI International, Menlo Park (États-Unis), avril 2009.

OCDE, Sickness, Disability and Work. Breaking the barriers, OCDE, Paris, 2010. 
Pan Ké Shon J.-L., « Partir de chez ses parents de 1968 à aujourd'hui », Idées, n 162, 2010/4, p. 33-41.

Rick 0., Ebersold S. (dir.), Lycéen, et après ? Les parcours vers l'enseignement supérieur et l'emploi, INS HEA, Suresnes, 2011.

VAn de Velde C., Devenir adulte. Sociologie comparée de la jeunesse en Europe, Presses universitaires de France, coll. « Le lien social », Paris, 2008.

\section{LES AUTEURS}

\section{Philippe Cordazzo}

cordazzolunistra.fr

Maître de conférences en démographie, HDR, Université de Strasbourg, membre du laboratoire SAGE (Société, acteurs, gouvernement en Europe, UMR 7363).

Thèmes de recherche : populations vulnérables; enseignement supérieur ; trajectoires professionnelles et entrée dans la vie adulte.

\section{A notamment publié}

CoRdazzo P., « Parcours étudiants : de la formation à l'insertion professionnelle », habilitation à diriger des recherches (HDR), 2013 (consultable sur halshs.archivesouvertes.fr/tel-01077686).

CoRdazzo P., Rick 0., «Appréhender le devenir des jeunes adultes handicapés à l'issue de l'enseignement secondaire : enjeux et difficultés de mesure de la marge dans l'ordinaire », Populations vulnérables, Populations et handicap, n 1, 2013, p. 95-130.

Cordazzo P., Sembel N., « Du Master à l'aide sociale : déclassement immédiat, "piège statutaire", nouvelles trajectoires de précarisation », O.S.P. L'orientation scolaire et professionnelle, n 41/1, 2012.

\section{Serge Ebersold}

serge.ebersolddinshea.fr

Professeur de sociologie, INSHEA/Université Paris Lumières, membre du laboratoire SAGE (Société, acteurs, gouvernement en Europe, UMR 7363) et du GRHAPES (Groupe de recherche sur le handicap, l'accessibilité et les pratiques éducatives et de scolarisation, EA 7287).

Thèmes de recherche : handicap ; politiques sociales ; éducation des personnes vulnérables; comparaisons internationales.

\section{A notamment publié}

Ebersold S., La naissance de l'inemployable ou l'insertion aux risques de l'exclusion, Presses universitaires de Rennes, Rennes, 2001.

EBERSoLd S., Le temps des servitudes. La famille à l'épreuve du handicap, Presses universitaires de Rennes, Rennes, 2005.

EBersold S., Parents et professionnels face au dévoilement du handicap. Dire et regards, Toulouse, Eres, coll. « Connaissance de la diversité », 2007. 